\title{
The effects of ultrasonic stimulation on DP- bioglass bone substitute
}

\author{
Feng-Huei Lin, Chi-Chang Lin*, Chung-Ming Lu, Haw-Chang Liu* \\ and Cheng-Yi Wang
}

Center for Biomedical Engineering and *Department of Orthopaedics, College of Medicine, National Taiwan University, Taipei, Taiwan, ROC

Received 25 September: accepted 18 January 1994

\begin{abstract}
In previous studies, DP-bioglass showed good biocompatibility and can form a chemical bond with natural bone. After implementation in the rabbit femur condyle for 32 weeks, DP-bioglass gradually biodegraded and asteocytes grew into the material. In this study, an attempt has been made to utilize low intensity pulsed ultrasound to speed up the bone regeneration rate and DP-bioglass absorption rate when the DP-bioglass is implanted into the rabbit femur condyle as a bone substitute.

The fundamental parameters of the ultrasound used were $1.5 \mathrm{MHz}$ frequency, $0.5 \mathrm{~W} \mathrm{~cm}^{-2}$ intensity, on-off ratio 1:1 and 2 ms for the on-off time interval. The stimulation, in all cases, was started $24 \mathrm{~h}$ after the operations by applying the transducer to the skin using DIR ultrasound jelly as a coupling medium. The evaluation of the progress of bone regeneration and the material's biodegradable rate were conducted by histological examination and by measurements of the areas of regenerated bone, pores and DP-bioglass made with a planimeter. It was found that low intensity pulsed ultrasound had a profound effect on the rate both of bone regeneration and DP-bioglass bioabsorption in this rabbit model and that its mechanism of the action may be via an electromechanical kinetic effect on the cell membrane interfaces.
\end{abstract}

Keywords: DP-bioglass, bone substitute, ultrasonic stimulation

Med. Eng. Phys., 1995, Vol. 17, 20-26, January

\section{INTRODUCTION}

Electromechanical interactions are thought to regulate cellular proliferation, differentiation, and function. The sources of electrical energy reside intrinsically within the bone (standing potential); may result from stress applied to the bone, whereby extracellular components of bone, notably collagen, act as transducers to convert mechanical to electrical energy (stress-generated potential); or are derived from externally applied electric or time-varying electromagnetic fields ${ }^{1-3}$. The preliminary clinical data suggest that they may be of use in osteogenesis, bone grafting and osteoporosis $^{1}$. In the clinical field, externally applied electric currents have been proposed but despite their success have certain disadvantages":

1. Electrical stimulation by electrodes is invasive, exposing the patient to the risk of infection.

2. Electromagnetic induction, employing coils, is a non-invasive process but takes a long period

Correspondence and reprint requests to: Feng-Huei Lin PhD, Associate Professor, Center for Biomedical Engineering, College of Medicine, National Taiwan University, Taipei, Taiwan, ROC

(c) 1994 Butterworth-Heinemann for BES

$1350-4533 / 95 / 01020-07$ of time for the treatment $(8-12 \mathrm{~h}$ a day over 4-8 months).

Ultrasound has been an interesting subject of study in recent years due to wide ranging applications particularly in medical diagnosis. The reasons for its uses are twofold. Firstly, the action of ultrasound does not produce cumulative effects such as are inherent in, for example, x-rays or beams of high energy particles, and secondly, it can provide information regarding tissue structure that is not obtainable by any other diagnostic techniques. Furthermore, ultrasound has been used for bonding fractured bones, cutting live biological tissues and for stimulating tissue regeneration and bone growth and repair. A great advantage of ultrasonic stimulation of bone healing is the much shorter daily treatment time that is needed compared to electrical stimulation; only 10-20 min a day over 2-8 months ${ }^{4}$.

In the previous study, DP-bioglass showed good biocompatibility and the ability to form a chemical bond with natural bone. The compositional profile of SEM-EPMA confirmed that ion inter-diffusion occurred at the interface of DP-bioglass 
and bony tissue. The formation of a reaction layer or diffusion zone was confirmed on the surface of DP-bioglass. The biological hydroxyapatite crystals were observed on the bony tissue and the layer, which was necessary for the DP-bioglass to bond directly with bone. After implantation in the rabbit femur condyle for 32 months, DP-bioglass was gradually biodegraded and osteocytes grew into the material which was revealed by histological evaluation $^{5-7}$. After a long period of implantation, the bioglass would be expected to be completely replaced by regenerated bone and become a part of the bone structure.

The metabolic rate of the rabbit is much higher than that of human. The DP-bioglass may be expected to be totally replaced by the bone structure in rabbit in an estimated 3 or 4 years, compared with 7 or 8 years in the human body; this has limited the use of DP-bioglass in clinical applications where it is hard to compete with other biodegradable bone grafts because of the longterm recovery. The main purpose of the present study was to focus on speeding up the biodegradation rate of DP-bioglass to better fit the demand of the clinical application. Ultrasonic stimulation was directly applied to the rabbit femur condyle where the defect cavity had been created and DPbioglass implanted as a bone substitute. It was the hope that the ultrasound would not only stimulate vigorous bone regeneration but, concurrently, would step up resorption and degradation of DPbioglass which would eventually disappear from the implantation site. The present report deals with the histological observations made to evaluate the effect of ultrasonic stimulation on DP-bioglass implanted in the rabbit femur condyle.

\section{MATERIALS AND METHODS}

\section{Preparation of DP-bioglass disc}

The glass used in the study was based on the DPbioglass of $\mathrm{Na}_{2} \mathrm{O}-\mathrm{CaO}-\mathrm{SiO}_{2}-\mathrm{P}_{2} \mathrm{O}_{5}$ system. A batch mixture of the nominal composition of $\mathrm{Na}_{2} \mathrm{O}$ $8.4 \% \mathrm{CaO} 40 \%, \mathrm{SiO}_{2} 39.6 \%$ and $\mathrm{P}_{2} \mathrm{O}_{5} 12 \%$, by weight was melted in a platinum crucible at $1400^{\circ} \mathrm{C}$ for $1 \mathrm{~h}$. The molten glass was poured into ice water at $0^{\circ} \mathrm{C}$ to quench as glass 'frit'. The glass 'frit' was pulverized into grains of about $5 \mu \mathrm{m}$ using a spex 8000 alumina ball mill. The glass powder, mixed with polyethylene glycol 4000 with particle sizes of 5 and $500 \mu \mathrm{m}$ to produce macroand micro-pore structures after decomposition, was pressed into discs of $6 \mathrm{~mm}$ in diameter and $5 \mathrm{~mm}$ in thickness under a hydrostatic pressure of $270 \mathrm{MPa}$. The glass powder compact was placed on a platinum sheet and heated to $750^{\circ} \mathrm{C}$ at a ratc of $5^{\circ} \mathrm{C} \mathrm{min}-1$ in a $\mathrm{SiC}$ heating element furnace, and then allowed to cool in the furnace after $2 \mathrm{~h}$ soaking time. The sintered porous DP-bioglass discs were then available for the rabbit femoral lateral condyle implants $s^{3,6}$.

\section{Operation and implantation}

In the experiment, ultrasonic energy was applied on the implanted area of 30 rabbits. Bilateral holes $(5 \mathrm{~mm}$ diam) were created in the outer condyle of the femur though the drill did not pass through the whole bone. No post-operation fracture was observed. The fundamental parameters relating to the applied ultrasound were $1.5 \mathrm{MHz}$ insonation frequency, $0.5 \mathrm{~W} \mathrm{~cm}^{-2}$ intensity, onoff ratio $1: 1$ and $2 \mathrm{~ms}$ for the on-off time interval ${ }^{4}$.

The stimulation, in all cases, started $24 \mathrm{~h}$ after the operations by applying the transducer to the skin using DIR ultrasound jelly as a coupling medium, right over the operation site. The acoustic impedance of the medium jelly was similar to that of skin $\left(1.5 \times 10^{-4} \mathrm{~g} \mathrm{~cm}^{-1} \mathrm{~s}^{-1}\right)$. The general procedure of each animal was as follows: (1) Ultrasonic stimulation was conducted on the left femur condyle for $15 \mathrm{~min}$ per day. (2) The right side received no ultrasound and was used as a control. A dummy transducer was applied to the contralateral limb, also using jelly. The animals were harvested at 1, 2, 3, 4, 6 and 8 weeks postoperatively. Five animals were killed at each experimental stage.

The 30 experimental rabbits were intramuscularly injected with oxytetracycline $\left(30 \mathrm{mg} \mathrm{kg}{ }^{1}\right)$ and subcutaneously injected with calcein green $\left(15 \mathrm{mg} \mathrm{kg}^{-1}\right)$, which was injected alternately every other week as a bone regeneration labelling stain $^{t .8}$. Specimens were subsequently examined using fluorescent microscopy.

\section{Measurements and analysis}

Evaluation of the progress of bone regeneration and the material's degradation rate were performed by histological examination and by measuring the areas of regenerated bone, DP-bioglass and pores. To measure the areas of interest, the bones were excised from the animal bodies, cleaned of soft tissuc, photographed and mcasured with a planimeter.

The excised bone specimens, from which any remaining soft tissue had been removed, were fixed in $40 \%$ methanol for $24 \mathrm{~h}$. For the polymethacrylate embedding to be successful, the embedding materials must be able to penetrate the bone specimen completely. This required thorough dehydration and lipid extraction of the fixed-bone specimens, performed in a graded alcoholic series, for $24 \mathrm{~h}$ in each solution. The thin sections, $0.2 \mathrm{~mm}$ in thickness, were cul on a milling machine with a diamond saw cutting perpendicular to the axis of the disc of the material. The sections were ground on a grinding machine with graded SiC-paper and polished with a diamond lappins disc to $30 \mu \mathrm{m}$ for histological evaluation under light and fluorescence microscopy $y^{5}$.

Non-invasive techniques are currently unavailable for the evaluation of bone growth into porous implants. Histomorphometric quantification of bone regeneration in the porous DPbioglass revealed an average bone, implant and soft tissue area fraction. Quantitative evaluation was performed by a semi-automatic histomorphometric method. The system consisted of a microscope with cross-polarization filters, digitizing platen, digitizer and microcomputer. The micro- 


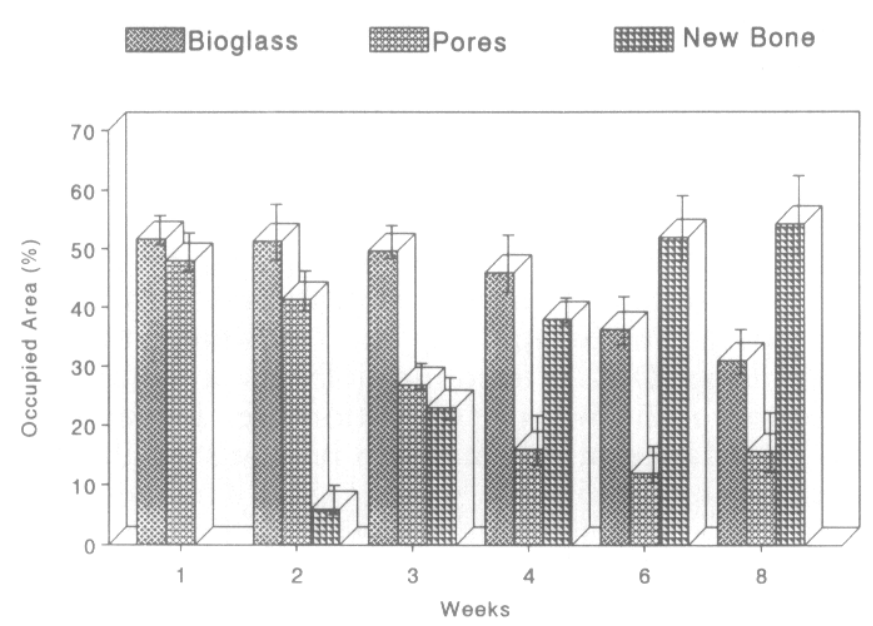

Figure 1 The summarized results of ultrasonic stimulation on the DP-bioglass implanted into the rabbit femur condyle. Series 1, area of DP-bioglass; Series 2, area of pores; Series 3, area of new bone

scope was equipped with a draw tube, through which the image of the digitizing platen was projected over the optical field. By moving a cursor on the digitizing platen, which was visiblc by its projection over the histological field, newly grown bony tissue in the defective cavity was traced, and its area calculated by a microcomputer. The area of newly grown bone structure in each section was calculated, and expressed as a percentage of the area of the created cavity defect ${ }^{9}$.

\section{RESULTS AND DISCUSSION}

The amount of newly grown bone, implants (DPbioglass) and pores were expressed as pcrcentagcs of their area compared to the area of the defect cavity (created hole); the area of defect cavity was defined as $100 \%$. The area occupied by pores included areas of soft tissue, connective tissue, bone marrow, osteoid and pores, etc.

The quantitative assessment of bone regeneration, pores and DP-bioglass, taken with a planimeter, were summarized in Figures 1 and 2 for ultrasonic stimulated bone and unstimulated (control) bone, respectively. The bone regener-

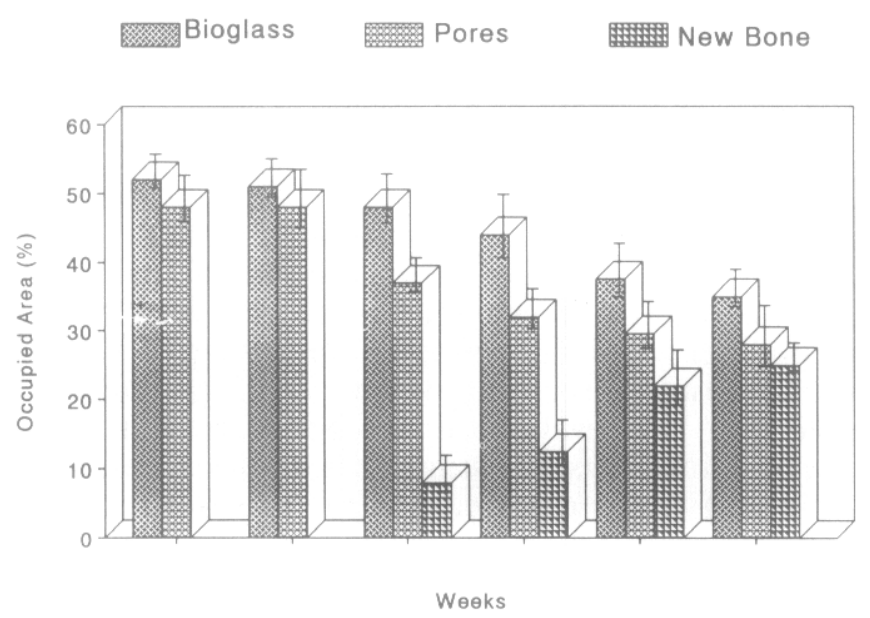

Figure 2 The summarized results of the histological observation without ultrasonic stimulation on the DP-bioglass implanted into the rabbit condyle. Series 1, area of DP-bioglass; Series 2, area of pores; Series 3 , area of new bone ation and biodegradation rate of the DP-bioglass showed a significant difference between the ultrasonic stimulation and unstimulated groups at the different experimental time periods. The former showed a higher rate of DP-bioglass degradation and new bone growth than those of the latter.

Figure $3 a$ and $b$ represents the results of the histological investigation of ultrasonic stimulation and non-stimulation, respectively, after DP-bioglass implantation for 1 week. The surface of the DP-bioglass was covered with a layer of fibrous tissue without any inflammatory reaction. There was no meaningful difference between the two groups in this stage.

Figure $4 a$ and $b$ show the results of histological sections from the ultrasonic-stimulated and unstimulated bone, respectively, at the 2 nd week after operation. In Figure $4 b$, the unstimulated pictures showed no regeneration bone around the DP-bioglass. The stimulated ones have already exhibited the osteoid across the inter-pores and newly grown bone directly altached onto the surface of the DP-bioglass (as shown in Figure $4 a$ ). There is no evidence of abnormal neoformation, a fact that was observed in all histological sections throughout the experimental periods.

New bone formation began to increase in the ultrasonic stimulation group 3 weeks after the operation. The areas of bone regeneration, pores
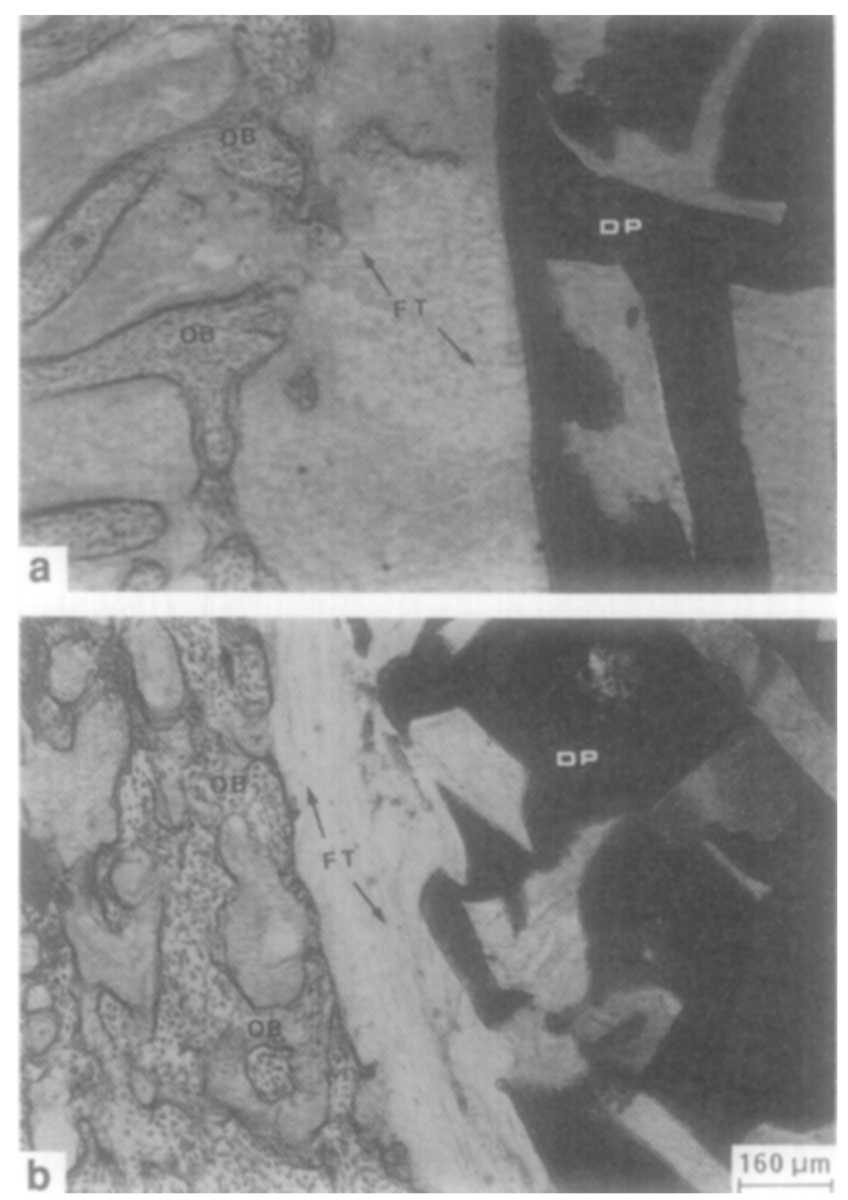

Figure 3 Microphotographs of the histological investigation of $a$, ultrasonic stimulation group and $b$, control group, after DP-bioglass implanted for 1 week. $\mathrm{FT}=$ fibrous tissue, $\mathrm{DP}=$ bioglass, $\mathrm{OB}=$ old bonc. $\mathrm{Bar}=160 \mu \mathrm{m}$ 

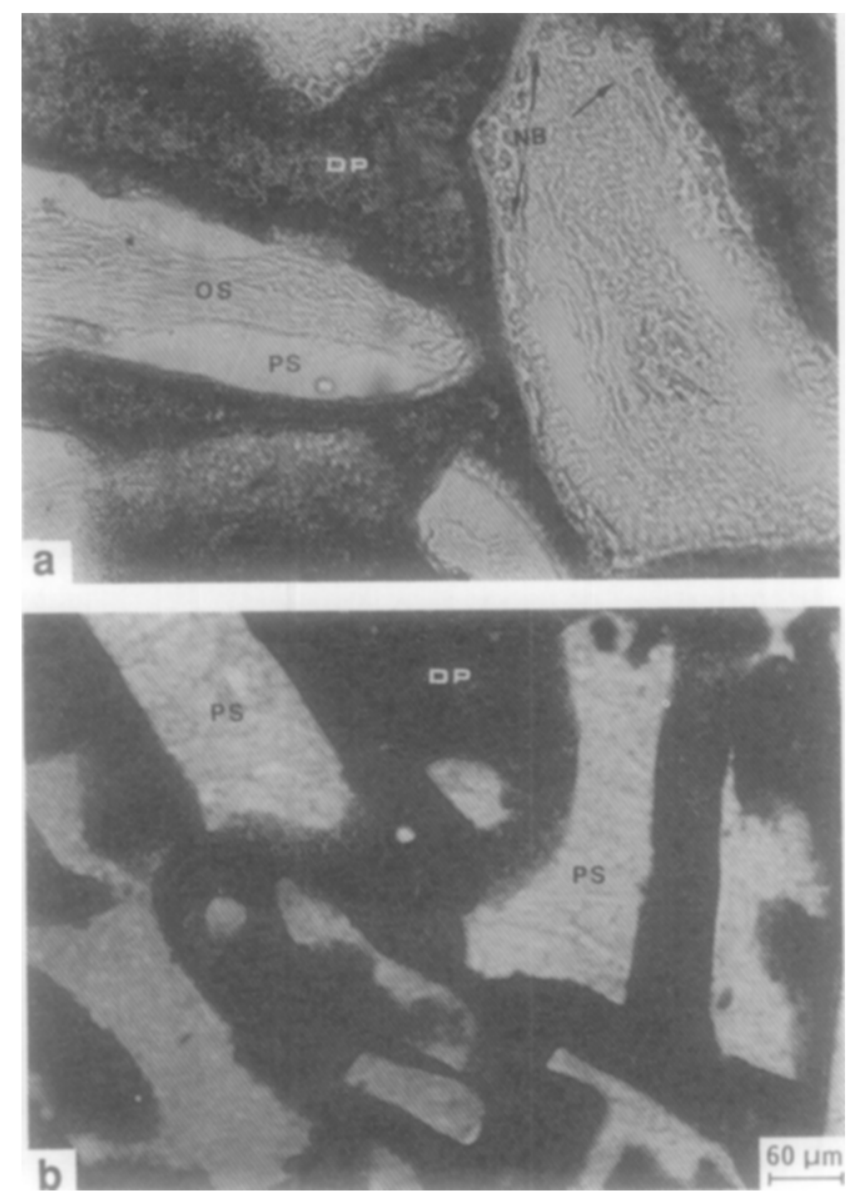

Figure $4 a$, Histological section of ultrasonic stimulated group a the 2nd postoperative week. Osteroid was obsened across the interpores of the materials and the new generation bone wats deposited on the surface of the DP-bioglass. $b$. The picture represented the section of the unstimulated group at the Ind week alter operation. The fibrous tissue has disappeared but not much has changed for the material. $D P=$ DP-bioglass, $N B=$ new bone $O S=$ osteroid, $P S=$ pores. $B a r=60 \mu m$

and DP-bioglass of the stimulated group were measured as $23 \%, 27 \%$ and $50 \%$, respectively, at the 3rd postoperative week. The unstimulated group, however, were estimated at $7 \%, 41 \%$ and $52 \%$. Figure $5 a$ and $b$ shows that the quantity of the new bone growth in the stimulated group was much higher than that in the unstimulated group. The phenomenon of DP-bioglass absorption in the in-vivo environment had already started for the stimulated group following implantation for 3 weeks, but was still unchanged in the controlled group.

At the 4th postoperative week, histological sections (Figure 6) show the stimulated group overhauling the unstimulated one, not only in terms of bone regeneration and DP-bioglass absorption rate, but also in the appearance of laminar bone structures and Haversian systems which are the evidence of the on-going bone remodelling process. There was no laminar bone to be observed in the sections of the unstimulated group at this stage. Figure 7 shows the closed examination of the ultrasonic treatment (stimulated) group 4 weeks after operation. The Haversian system and mature laminar bone can be clearly observed at this stage.
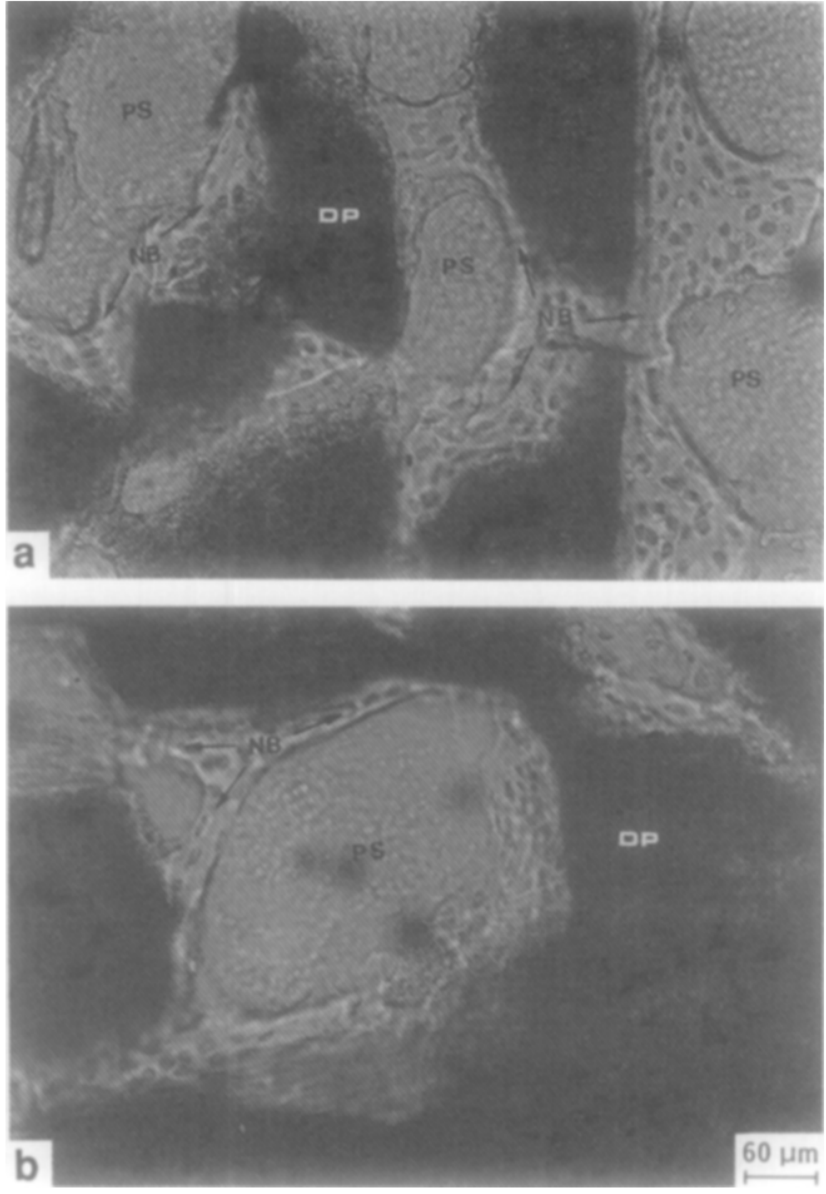

Figure $5 a$, The section of the stimulated group showed a vigorous bone regeneration and the primary bone grew into the macropores of the materals at the 3ad seek after the operation. b, Microphotograph show the metimulated group at this stage. The DP-bioglass degradation late and bone regeneration rate were much lower than that of the stimulated group. $\mathrm{DP}=\mathrm{DP}$-bioglass, $\mathrm{NB}=$ uew bone,

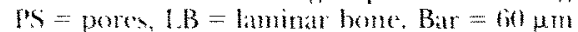

Six weeks after the operation, vigorous bone remodelling was observed everywhere in the ultrasonic treatment group (as shown in Figure $8 a$ ). In Figure $8 b$, no evidence of the bone remodelling process was proved for the unstimulated group, nor indeed of the laminar bone structures. The same scenario was demonstrated in the sections taken at the 8 th postoperative week. Figures 9 and 10 are fluorescent microphotographs of the stimulated and unstimulated groups, respectively. The pictures illustrate the quantity of the new bone growth every week which were labelled by calcine green and oxytetracycline. The stimulated yroup reveals a broader space between the labelled stripe, as shown in Figure 9. Figure 10 showing the unstimulated group, however, showed a narrower space between each stripe line. The results imply that the new bone growth of stimulated group was much more vigorous than that of unstimulated group. The summarized data are displayed in Fig ures 1 and 2. The data show that the ultrasonic treatment in this experiment could induce bone regeneration, speed up the bone remodelling process and enhance DP-bioglass bioabsorption in the physiochemical environment.

The above data show that ultrasonic stimulation 

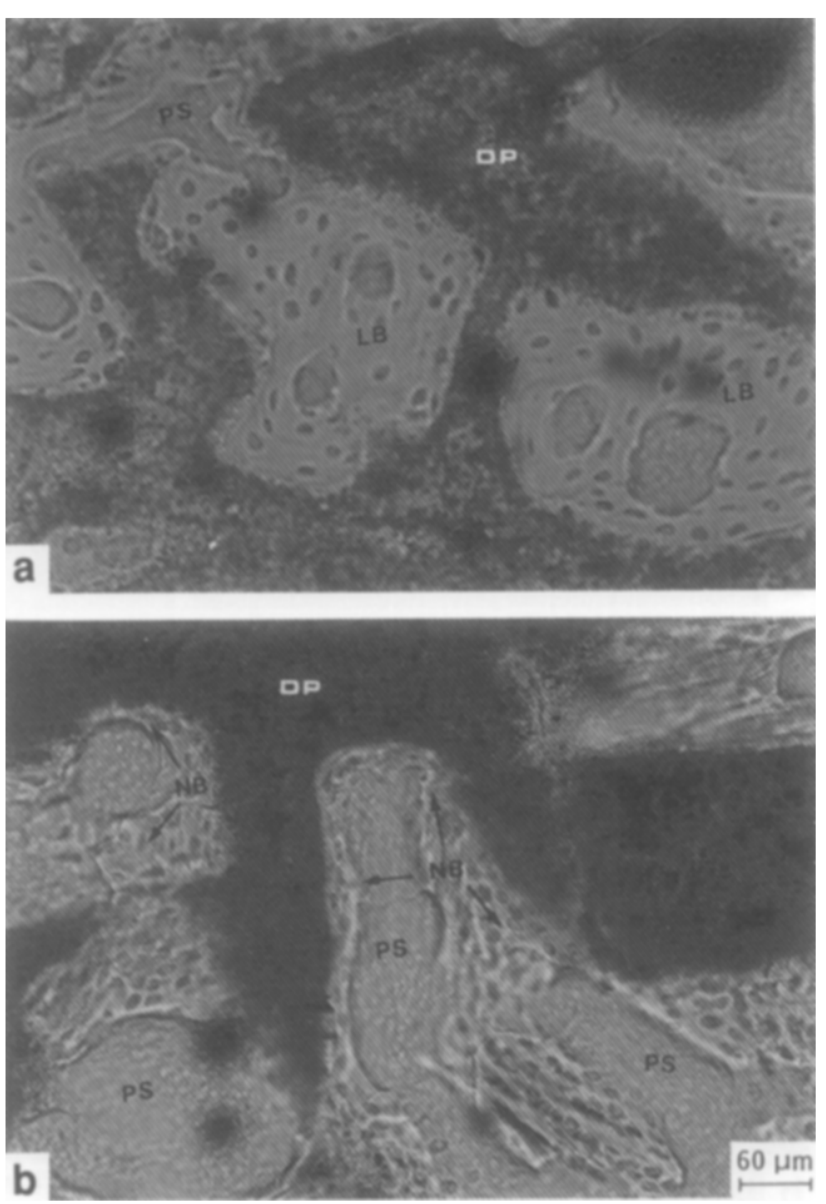

Figure 6 At the 4th postoperative week, the histological sections showed that $a$, stimulated bone was overhauling $b$, unstimulated bone in regeneration and DP-bioglass absorption rate. "The bioglass was gradually replaced by the new bone and laminar appeared in the section of stimulated group at this stage. DP = DP-bioglass, $\mathrm{PS}=$ pores, $\mathrm{LB}=$ laminar bone. $\mathrm{Bar}=60 \mu \mathrm{m}$

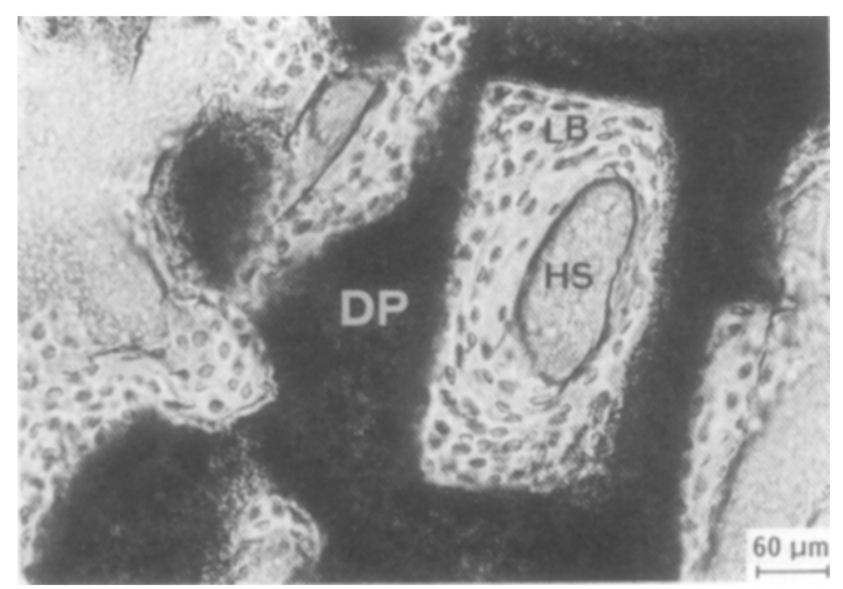

Figure 7 Haversian system and laminar bone have developed in the stimulated group at the 4 th week after operation. $\mathrm{HS}=$ Haversian system, $\mathrm{LB}=$ laminar bone, $\mathrm{DP}=\mathrm{DP}$-bioglass. $\mathrm{Bar}=60 \mu \mathrm{m}$

can successfully induce osteogenesis to rapidly increase bone regeneration during the healing process surrounding DP-bioglass implanted as a bone substitute into the rabbit femur condyle, and can speed up DP-bioglass degradation (or absorption) rate. By applying the concept of Bassett $^{8,10}$, when an intact long bone is subjected to
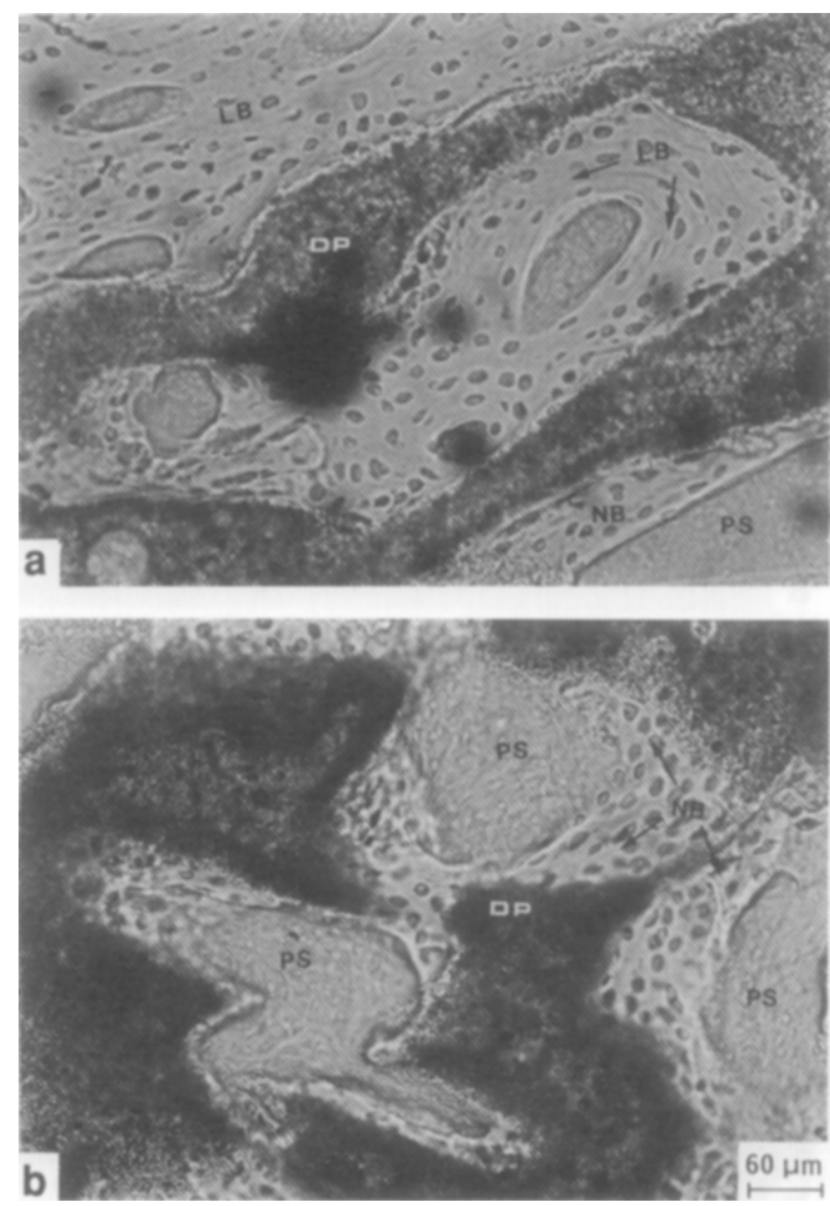

Figure 8 Histological observations of $a$, stimulated and $b$, unstimu lated bone at the 6 th week after operation. Bar $=60 \mu \mathrm{m}$

a compressive force, the periosteum can be induced to proliferate and form new bone. It appears that the metabolism of the cambium cells is affected by the mechanical energy, and from electrophysiological point of view, the biologically hyperactive tissue becomes electronegative. Since force generates electricity equally as well in either viable or nonviable bone, the sequence of biological events appears to be: force firstly generates electricity which then modifies bone cell activity. Consequently, chemical, thermal, or mechanical stimuli, which are capable of producing nonfracture callus, have been hypothesized to constitute 'the environmental condition which must first be converted to electrical energy which, in turn, acts upon the cells to cause them to proliferate'1!. The ultrasonic stimulation applied in this present investigation served as a source of external force or mechanical stress to produce the electrical energy and streaming potentials needed to favour bone accretion rate and to increase blood supply which in turn leads to the encouraging results in this study.

The actual mechanisms by which the application of any form of energy induces osteogenesis are not yet known. Proposed mechanisms centre around direct and indirect mechanical effects. With respect to the direct mechanical effect, it has been reported that bone cells respond to static mechanical forces by an increase in prostaglandin 

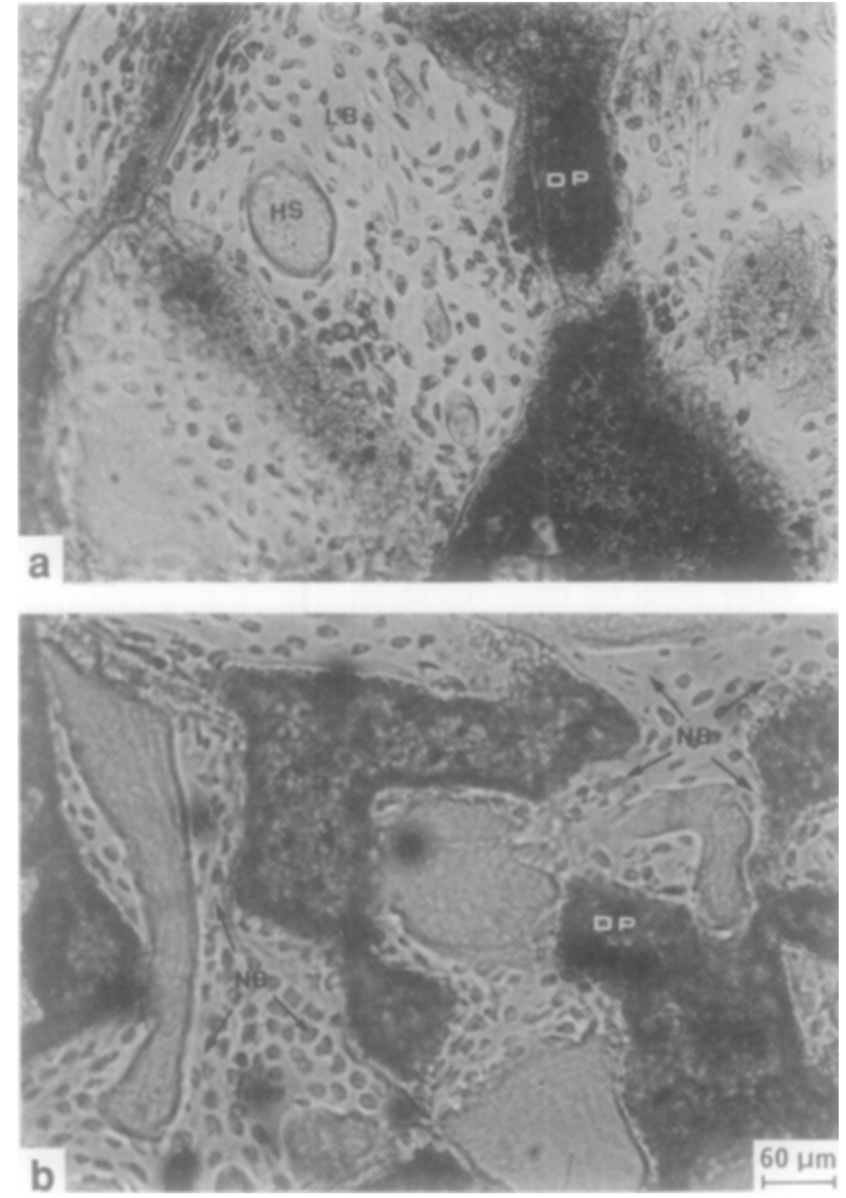

Figure 9 Histological observations of $a$, stimulated and $b$, unstimulated bone at the 8 th week after operation. Bar $=60 \mu \mathrm{m}$

$\left(\mathrm{PGE}_{2}\right)$ synthesis, and this is a direct result of the deformation of membrane phospholipids. PGE has long been known to be the most potent bone absorbing agent and to stimulate bone formation; other studies showed that $\mathrm{PGE}_{2}$ activated osteoclast-like cells in vitro ${ }^{12-15}$. These studies along with our results, would suggest the possibility that the ultrasonic stimulation effect on bone regeneration, remodelling and DP-bioglass absorption observed in this study is mediated by the osteoblast via $\mathrm{PGE}_{2}$ production and subsequent $\mathrm{PGE}_{2}$ action on the osteoclast. Ultrasound propagates through the biological medium (tissue) causing pressure and temperature changes ${ }^{16}$. Negligible temperature changes occur in this system because of the low incident power. The pressure changes are caused by compression and rarefaction of the fluid and cells. These rapidly changing mechanical forces $(1.5 \mathrm{MHz})$ may cause vibrations (deformations) of the cell membrane or extracellular matrix. A direct mechanical deformation of the membrane may alter ionic permeability, and therefore second messenger activity.

It is possible, however, that ultrasonic stimulation may cause indirect mechanical effects which act via a pathway similar to that thought to exist for electrical stimulation ${ }^{3,4,10}$. Here, the cell membrane appears to be the target, with various second messenger systems proposed as the initiators of the biological response. Adenyl
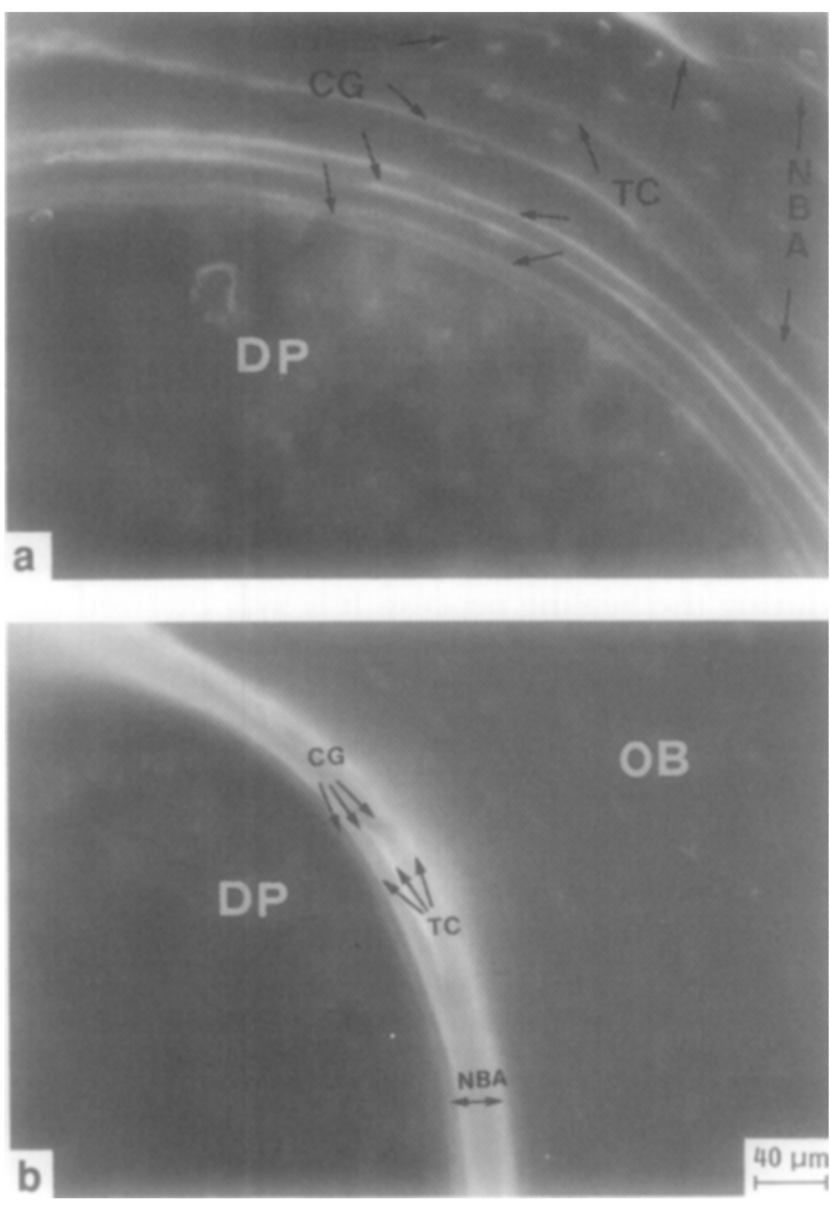

Figure 10 Fluorescent microphotographs of $"$ stimulated and $b$ unstimulated bone at the sth week after operation. $\mathrm{NBA}=$ new bone area, $\mathrm{DP}=\mathrm{DP}$-bioglass, $\mathrm{T}_{\mathrm{C}}=$ oxvtetacycline, Cg $=$ calcine green. $\mathrm{B}_{\mathrm{ar}}=40 \mu \mathrm{m}$

cyclase is a hormone receptor within the membrane and is subject to the influence of various microenvironmental factors (e.g. local ionic concentrations, mechanical pressures that distort the cell membrane), which in turn may affect the electrostatic potential of the plasma membrane. Many polypeptide hormones carry out their function in specific target tissues by increasing the adenyl cyclase activity, within the chosen cell membrane, and increasing the intracellular level of cyclic AMP ${ }^{17.19 .26}$. In bone cells, parathyroid hormone and calcitonin, by interaction with adenyl cyclase, will modulate the cyclic AMP level within the bone cells, and thus effect remodelling. The intracellular levels of the cyclic nucleotides cyclic AMP and guanosine 3',5'-monophosphate (cyclic GMP) also play a role in cytodifferentiation and cell division. At the intracellular level in bone, cyclic AMP and cyclic GMP are regarded as the mediators of extrinsic factors acting on the cell membrane to induce osteogenesis ${ }^{15}-80$. Therefore, any perturbation that produces a change in potential across the interface will alter the specific interactions of charged species in the extracellular fluid with, for example, enzymes or steroid hormones, resulting in a modification of cell function. The frequency of the pressure wave generated from the ultrasound is well within the response time for movement of ions at the surface 
of the cell membrane of the macromolecules in the extracellular matrix. Modulation of, for example, ion binding at the membrane surface due to pressure-induced ion movement is an electrochemical perturbation which may be caused by the mechanical input. These may directly relate to the basic acceleration of bone regeneration, remodelling and DP-bioglass degradation observed in this study.

\section{CONCLUSION}

Ultrasonic stimulation can successfully be applied to DP-bioglass bone grafts and have a great improvement in the material's absorption speed during the healing process. It is hypothesized that the significant difference between stimulated and unstimulated group in the bone regeneration as well as the DP-bioglass degradation rate in this experiment were a result of the ultrasound stimulation-induced electrical energy affecting bone cell metabolism, and activating osteogenesis and osteoclast activity at the implantation site.

\section{ACKNOWLEDGEMENT}

The investigations were supported by grant No. NSC-81-0412-B002-76 of National Sciences Council, Republic of China.

\section{REFERENCES}

1. Zor U, Kaye, AM, Shimshoni Z, Harell A, Somjen D. The transduction of mechanical force into biomechanical events in bone cells. Calcif Tissue Int 1988; 42: 261-6.

2. Behari J, Singh S. Ultrasound propagation in in-vivo bone. Ultrasonics 1981; March: 87-90.

3. Klug W, Franke WF, Knoch HG. Scientific control of bone-fracture healing under ultrasonic stimulation. Eur J Nucl Med 1986; 11: 494-7.

4. Chang WH, Liu HC, Liu TK. Clinical evaluation of bone fracture healing enhanced by electrical stimulations. $J$ Chinese Biomed Eng Soc 1991; 12: 125-8.
5. Lin FH, Huang YY, Hon, MH, Wang CY. Fabrication and biocompatibility of a porous bioglass ceramic in a $\mathrm{CaO}-$ $\mathrm{Na}_{2}-\mathrm{SiO}_{2}-\mathrm{P}_{2} \mathrm{O}_{5}$ system. J Biomed Eng 1991; 13: 328-34.

6. Lin FH. Preparation and characterization of highstrength bioglass ceramics composed of $\mathrm{Na}_{2} \mathrm{O}-\mathrm{CaO}-\mathrm{SiO}_{2-}$ $\mathrm{P}_{2} \mathrm{O}_{5}$ system. $J$ Chinese Biomed Eng 1992; 12: 339-408.

7. Lin $\mathrm{FH}$, Hon MH. A study on the structure of $(\mathrm{Na}, \mathrm{Ca})$ $(\mathrm{P}, \mathrm{Si}) \mathrm{O}_{3}$ crystallized in the $\mathrm{NaO}_{2}-\mathrm{CaO}-\mathrm{SiO}_{2}-\mathrm{P}_{2} \mathrm{O}_{5}$ glass. $J$ Jpn Ceram Soc 1990; 98: 1-6.

8. Bassett CAL. Pulsing electromagnetic fields: A new method to modify cell behavior in calcified and non-calcified tissues. Calcif Tissue Int (1982); 34: 1-8.

9. Ono K, Yamamuro T, Nakamura T. Mechanical properties of bone after implantation of A-W containing glass ceramic-fibrin mixture. $J$ Biomed Mater Res 1990; 24: 47 63.

10. Bassett CAL. Biological significance of piezoelectricity. Calcif Tissue Int 1968; 1: 252-30.

11. Friedenberg $7 B$, Brighton CT. Bioelectrical potentials in bone. J Bone Joint Surg 1966; 48A: 915-21.

12. Binderman I, Zor U, Kaye AU, Shimshoni Z, Harell A, Somjen $\mathrm{D}$. The transduction of mechanical force into biochemical events in bone cells may involve action of phospholipase $A_{2}$. Calcif Tissue Int 1988; 42: 261-6.

13. Tataki DN, Schneeberger G, Dziak R. Recombinant interleukin-1 stimulates prostaglandin $\mathrm{E}_{2}$ production by osteoblastic cells: synergy with parathyroid hormone. $\mathrm{Cal}$ cif Tissue Int 1988; 42: 358-62.

14. Somjen D, Binderman I, Berger E, Harell A. Bone remodelling induced by physical stress is prostaglandin mediated. Biochem Biophys Acta 1980; 627: 91-100.

15. Somjen D, Kaye AM, Binderman I. Stimulation of creatine kinase $\mathrm{BB}$ activity by parathryoid hormone and by $\mathrm{PGE}_{2}$ in cultured bone cells. Biochem J 1985; 225: 591-6.

16. Pilla AA, Figueiredo M, Nasser P. Pulsed sine wave ultrasound accelerates bone fracture repair. Ultrasonic Symposium 1989; 1003-5.

17. Graham F, Pounds D. Transient cavitation in tissue during ultrasonically induced hyperthermia. Med. Phys. 1982; 9: $1-3$.

18. Chang JZ. Study on the mechanism of enchancing callus formation of fracture by ultrasonic stimulation. J Chinese Biomed Eng Soc 1992; 11: 120-4.

19. Pilla AA. Dynamic electrochemical phenomena at living cell membranes. J Electrochem Soc 1975; 122: 126c.

20. Rodan GA. Cyclic AMP and cyclic GMP: mediators of mechanical effects on bone remodelling. Science 1975; 189: $467-70$. 